\title{
Visual Control Of Unmanned Aerial Vehicle For Handling Disaster Manangement Issue
}

\author{
Mr.Mayur S. Malokar \\ M.Tech Electronics and Telecommunication Government College of Engineering, Jalgaon, India \\ Corresponding Author: Mr.Mayur S. Malokar
}

\begin{abstract}
In order to get the information of any vehicle which is travelling on the way we required to rely on Global Positioning System. The GPS provides the Global Positioning Information of vehicle, which relies on external source called satellites. But the satellite signal may get cut or have small signal strength in cluttered areas or is less reliable at low altitude areas, so I think of to use the Unmanned Aerial Vehicle. The main objective is to use the Raspberry pi to capture the stream of video to monitor and to detect the color or objects. Raspberry pi is a credit size single cheap computer in order to monitor the object. In order to make it explosive detection an IED sensor can be placed. (Due to non-availability of sensor not attached). The general purpose pins of Raspberry pi connected to direction control of the remote with help of servo motors in order to follow the color or object. It plays a great role in disaster occurred areas in order to detect the human beings or finding the objects. In a flooded area in order to monitor or detect the humans it is easy for UAV to move above the surface of the ground and water. And provides the detection as well as live streaming of the particular area and images can be captured by it. It is used to monitor the construction site, coal mine and much such monitoring application.
\end{abstract}

Date of Submission: 07-07-2017

Date of acceptance: 15-07-2017

\section{Introduction}

An unmanned aircraft system is just that - a system. It must always be considered as such. The systemComprises a number of sub-systems which include the aircraft (often referred to as a UAV or unmannedair vehicle), its payloads, the control station(s) (and, often, other remote stations), aircraft launch andrecovery sub-systems where applicable, support sub-systems, communication sub-systems, transportsubsystems, etc.It must also be considered as part of a local or global air transport/aviation environment with its rules, regulations and disciplines. UAS usually have the same elements as systems based upon manned aircraft, but with the airborneelement, i.e. the aircraft being designed from its conception to be operated without an aircrew aboard.The aircrew (as a sub-system), with its interfaces with the aircraft controls and its habitation is replacedby an electronic intelligence and control subsystem.

The other elements, i.e. launch, landing, recovery, communication, support, etc. have their equivalentsin both manned and unmanned systems. Unmanned aircraft must not be confused with model aircraft or with 'drones', as is often done bythe media. A radio-controlled model aircraft is used only for sport and must remain within sight of theoperator. The operator is usually limited to instructing the aircraft to climb or descend and to turn to theleft or to the right.A drone aircraft will be required to fly out of sight of the operator, but has zero intelligence, merelybeing launched into a pre-programmed mission on a pre-programmed course and a return to base. It doesnot communicate and the results of the mission, e.g. photographs, are usually not obtained from it untilit is recovered at base. A UAV, on the other hand, will have some greater or lesser degree of 'automatic intelligence'.It will be able to communicate with its controller and to return payload data such as electro-opticor thermal TV images, together with its primary state information - position, airspeed, heading andaltitude. It will also transmit information as to its condition, which is often referred to as 'housekeeping data', covering aspects such as the amount of fuel it has, temperatures of components, e.g. engines or electronics. If a fault occurs in any of the sub-systems or components, the UAV may be designed automatically to take corrective action and/or alert its operator to the event. In the event, for example, that the radio communication between the operator and the UAV is broken, then the UAV may be programmed to search for the radio beam and re-establish contact or to switch to a different radio frequency band if the radio-link is duplexed. A more 'intelligent' UAV may have further programmed which enable it to respond in an 'if that happens, do this' manner. For some systems, attempts are being made to implement on-board decision-making capability using artificial intelligence in order to provide it with an autonomy of operation, as distinct from automatic decision making. The definition of UAS also excludes missiles (ballistic or homing). The development and operation of UAS has rapidly expanded as a technology in the last 30 years and, as with many 
new technologies, the terminology used has changed frequently during that period. The initials RPV (remotely piloted vehicle) were originally used for unmanned aircraft, but with the appearance of systems deploying landbased or underwater vehicles, other acronyms or initials have been adopted to clarify the reference to airborne vehicle systems. These have, in the past, included UMA (unmanned air vehicle), but the initials UAV (unmanned aerial vehicle) are now generally used to denote the aircraft element of the UAS. However, UAV is sometimes interpreted as 'uninhabited air vehicle' in order to reflect the situation that the overall system is 'manned' in so far as it is not overall exclusively autonomous, but is commanded by a human somewhere in the chain. 'Uninhabited air vehicle' is also seen to be more politically correct! More recently the term UAS (unmanned aircraft system) has been introduced. All of the terms: air vehicle; UAV; UAV systems and UAS will be seen in this volume, as appropriate, since these were the terms in use during its preparation.

The earlier system were used for Military Application and the system was too big and were operated with a base station which require a large setup. The earlier system used were only used for viewing the live picture. It was not able to detect the particular object if required.

The system I am going to design is small in size and operated with remote control and Autonomously that means it work in auto mode in order to detect the object. I am going to detect the object with the help of Rispberry pi depending upon the motion that is motion detection object recognition. I am also making it to detect the explosive material with the help of IED sensor as per the availability of sensor. I am making the UAV able to used in the Disaster Management in order to detect the object. It also have functionality to follow a particular object continuously with given Instruction.

\section{Litreature Review}

UAVs have been in production since before the Wright Brothers first took their historic flight. The earliest account can be traced back to the American Civil War, when an inventor patented an unmanned balloon that carried explosives that could be dropped after a time-delay fuse mechanism triggered a basket to overturn its contents [2]. While this is a relatively primitive idea of what the world has come to know today as "drones" it goes to show how early man began thinking about unmanned aerial systems. This technology began taking small leaps in the years following the American Civil War - the first military aerial reconnaissance photos were taken in 1898 during the Spanish-American War via a camera attached to a kite [2]. As the trend shows, many of the advancements in this technology arose during times of war, whether it was used to help with an offensive strike or just to acquire intelligence on enemy locations and activities. This is seen throughout the history and progress of unmanned aerial technologies. Advancements took place in Britain during the 1930s, where a radiocontrolled UAV (dubbed the Queen Bee) served as aerial target practice for British pilots, and also during World War II, during which 10 time the Nazi's developed an unmanned flying bomber known as the V-1 [2]. It wasn't until the 1970s that Israel developed the Scout and the Pioneer, which started the development toward the more widely known glider-type UAVs [2]. It was from this design that the Predator drone came to be; the Predator is the most sophisticated UAV in existence to date, these drones have come a long way from the "balloons" of the past. It's autonomous control networks show just how much this technology has evolved.

The structural design of UAVs has changed over their developmental history in order to serve a variety of purposes. UAV design and advancement is a global activity. As technology and needs change, UAVs can be improved to serve these needs. There are several design considerations that are constant.

The first of these design criteria is the degree of autonomy. Early UAV designs were mostly set to fly a specified path until they ran out of fuel. They carried a camera onboard, which would be recovered after the UAV landed. Later, the advent of radio control systems allowed UAVs to be piloted from the ground. Modern UAVs often combine these two basic functionalities. These two modes of operation do not strictly signify autonomy. True autonomy suggests the ability of the aircraft to operate without human interaction. In this regard, UAVs are still very immature. UAV autonomy technology is divided into the following categories:

$>$ Sensor fusion: On board the vehicle a combination of sensors are used.

$>$ Communications: Communication and coordination will be handled between multiple sources in the existence of curtailed and imperfect information.

$>$ Motion planning (also called Path planning): Determining the optimal path for the vehicle in accordance with specific objectives and constraints such as obstacles go.

$>$ Trajectory Generation: Designed for optimal control and maneuverability to follow a particular route or to go from one place to another.

$>$ Task Allocation and Scheduling: Set the optimal distribution of tasks between a group of agents, with time constraints and equipment limitations.

$>$ Cooperative Tactics: The optimal sequence and spatial distribution of activities between agents in order to make the most of the chances of success in any case or situation. [3]

The ultimate goal of UAVs is to replace human pilots altogether. Another major design criterion is UAV endurance (range). Since there is no human pilot onboard, there is no concern for pilot fatigue. UAVs can 
be designed to maximize flight times to take advantage of this fact. Different systems can afford a wide variety of maximum range. Internal combustion engines require relatively frequent refueling and in-flight refueling is a major obstacle for this type of propulsion system. Photovoltaic UAVs offer the potential for unlimited range and there is much research in this field. One more type of fuel system is hydrogen, which is proposed for use with certain models of stratospheric persistent UAVs. The AeroVironment's Global Observer is one such UAV. This aircraft runs on hydrogen and has a range of 7 days. The idea is for two of such UAVs to be used in tandem to provide continuous, uninterrupted operation 365 days a year. [5]

With the sophistication that these systems have arrived at, the market for them has grown astronomically. While the United States still has the largest stockpile of unmanned aircraft, the rest of the world is beginning to follow suit. More than 50 countries have purchased surveillance drones, and many have started in-country development programs for armed versions [4]. More than two-dozen different models were shown at a recent aviation show in China [4]. Due to the changing landscape of the theater of war, many nations are leaning toward unmanned aircraft to handle delicate situations in which human lives need not be put at risk. Also, taking into account the fact that drones sell for a fraction of the cost of manned airplanes, the amount of UAVs a nation can purchase at once has enticed many nations into entering the drone zone.

In general, UAVs fall into one of six functional categories:

$>$ Target and Decoy: simulating enemy missiles or aircraft for ground and air gunnery

$>\quad$ Reconnaissance: battlefield intelligence gathering

Logistics: cargo and logistics application

$>$ Research and Development: used for UAV technology development

$>\quad$ Civil and Commercial: specifically designed for civil and commercial applications [6]

$>$ Competition details: The basis of the competition is a reconnaissance mission for the US Marines for specified target accusation as well as added fight directions mid-flight. The story is that an island has had storms and pirates have invaded the island. The UAV is to stay within a specified area and transmit the images back to base as well as locations for desired targets. [7]

Students are judged on how well the UAV performs the desired task and top teams receive prize money. The competition requires a submittal of a final journal paper, oral presentation, and demonstration of UAV capabilities. At the start of the competition a statement of work will be provided and it is the team's job to figure out the best system design and development to complete the task. [7]

A fact sheet will be needed by committee to review prior to competition of each plane to verify qualifications for the competition. Also well as the journal paper describing in detail each aspect of the plane with a detailed description of functions. The oral presentation is not an overview of the journal paper but rather a briefing of the plane and safety checks, Static checks, and testing development descriptions. Afterwards there will be a preflight brief done by safety inspectors. [7]

The flight demonstration will consist of taking off and landing in specified landing/takeoff zones as well as autonomous flight within given flight path, guided by GPS being able to stay out of no-fly zones. Targets should be recognized along this path and will be different geometric shapes, of different color and size. This mission should be able to be completed within 40 minutes and will receive extra points for any saved time down to 20 minutes. [7]

The requirements and parameters of the plane are a few to insure the safety of the competitors. The gross weight should not exceed 55 pounds. GPS location and flight height must be transmitted to judges at all times to ensure that plane is not in a no fly zone or altitude. There must be a manual override by the safety pilot in case of emergencies. The plane should have a return home activation if loss of signal for more than 30 seconds as well as being able to be activated by an operator. If signal is lost for more than 3 minutes a terminate flight system should be activated automatically by the plane as well should be able to be activated by the operator. The speed of 16 the plane should not exceed 100 KIAS. Batteries and plane should have some bright colors in the case of a crash the materials can be found easily. [7]

\section{$A$ PRINCIPLE OF QUADCOPTER AND ITS BASICS}

\section{System Concept And Flow}

A quadcopter is a four rotor unmanned aerial drone that operates with the same principle as of a helicopter with slight differences lying within both. It is used for several purposes in the military as well as social fields operating over a wide range of applications. It has become a craze for building a quadcopter among people as it gives a wide variety of benefits as compared to the traditional helicopter such as stability and control. Till date quadcopter has only been used as a drone that is controlled from a distant place.

A general purpose quadcopter is X-type quadcopter with every motor rotating in the anti-spin direction to that of its adjacent motor. The quadcopter works over two principles: 


\section{(i) Newton's Third Law of Motion}

In order to create a movement forward, the quad has to push the air backward. Thus for every backward motion of air, there is a forward motion of quadcopter.

\section{(ii) Bernoulli's Principle}

According to Bernoulli's theorem there is an increase in velocity if there is a decrease in pressure and vice versa. Hence in order to increase the velocity the pressure at that point needs to be decreased.

There are four basic movements of the quadcopter viz. throttle, elevator, aileron and rudder.

- Throttle is considered when there is an upward and downward motion of the quad. It is used for takeoff and landing of the quad. It is created by rotating all 4 rotors at the same speed.

- Elevator is considered when there is a forward and backward motion of the quad. In an X-type quad forward motion is created by rotating the 2 rear rotors at greater speed compared to the front rotors whereas for backward motion the front rotors have higher speed than rear rotors.

- Aileron is considered when there are right and left movements. It is used for changing directions in the moving drone. It is created by rotating the left 2 rotors to rotate at a higher speed than the right rotors for the right turn and vice versa for the left turn.

- Rudder action is used to create clockwise and anti-clockwise movement of the quadcopter about the central axis. It is achieved by rotating the diagonally situated rotors moving with the same spin to rotate at a greater speed than the other two.

Apart from the above operations, the quad can also use a GPS to track its own location.

For small scale building of quadcopters, BLDC motors are best because of their light weight, less friction and high speed. $1000 \mathrm{KVA}$ BLDC motors would be suitable in case of a quad with a dimension of $35 \times 35 \mathrm{x} 15 \mathrm{~cm}$. Unlike geared DC motors, BLDC motors cannot be used directly in the electrical circuit. To control the movement and speed of the motor an electronic speed controller (ESC) is used that signals the BLDC motor according to the data received by it. For each BLDC motor, there must be one ESC in order to run the motor.

The flight movements are caused by means of propellers. The size of propeller must be appropriate depending upon the size of the quad in order to create enough thrust to cause the movements.

The cheapest and the most efficient flight control board is the KK version 5.5. It is suitable for beginners. For high-level multipurpose applications, various other flight controllers are used. The most widely used flight controller is NAZZA M-Lite manufactured by DJI Company.

The powering unit of the system is a rechargeable lithium polymer battery. It is better suited because of its light weight and high current supply for the quad movements. Since the quadcopter is an unmanned aerial drone transmission of signals takes place wirelessly via radio frequency transmission system (RFTS). This is achieved by means of a 6 channel radio control transmitter and receiver.

\section{B CONSTRUCTION}

The four BLDC motors are mounted on the ends of the quad frame each connected to the flight control board via ESC. The 4 motors are connected in parallel to the supply battery. The ESCs are connected to their respective 4 pins M1, M2, M3 and M4 on the KK board. The receiver is then attached to the KK board. Four channels are used in the receiver for the four different movements and are connected to their respective pins namely the throttle, elevator, aileron and rudder pins. Only one of the pin pair is made in connection to the Vcc and Gnd pins and the remaining three are connected via signal pins. The KK board is an inbuilt microprocessor that gives out the desired output to the motors by receiving the signals for the different movements via the receiver.

\section{CWORKING PRINCIPLE}

- As discussed earlier there are 4 motions in quadcopter viz. throttle, elevator, aileron and rudder. These motions are controlled by the help on a radio control transmitter.

- The transmitter transmits the data to the receiver which is placed over the quad.

- The receiver then sends the data to the flight control board.

- The flight controller is an inbuilt microprocessor that manipulates the signals received by it and commands the BLDC motors through the ESCs.

- After the command is received the motors act according to the signal transmitted by the remote.

- The throttle is created by rotating all 4 rotors at the same speed.

- An elevator is created by rotating the 2 rear rotors at greater speed compared to the front rotors whereas for backward motion the front rotors have higher speed than rear rotors.

- Aileron is created by rotating the left 2 rotors to rotate at a higher speed than the right rotors for the right turn and vice versa for the left turn. 
- Rudder action is created by rotating the diagonally situated rotors moving with the same spin to rotate at a greater speed than the other two.

Table I shows the controlling of Yaw Roll and Pitch for stability

\begin{tabular}{|c|c|c|c|}
\hline $\begin{array}{c}\text { Primary Control } \\
\text { Surface }\end{array}$ & $\begin{array}{c}\text { Airplane } \\
\text { Movement }\end{array}$ & $\begin{array}{l}\text { Axes of } \\
\text { Rotation }\end{array}$ & Type of Stability \\
\hline Aileron & Roll & Longitudinal & Lateral \\
\hline Elevator/ Stabilator & Pitch & Lateral & Longitudinal \\
\hline Rudder & Yaw & Vertical & Directional \\
\hline
\end{tabular}

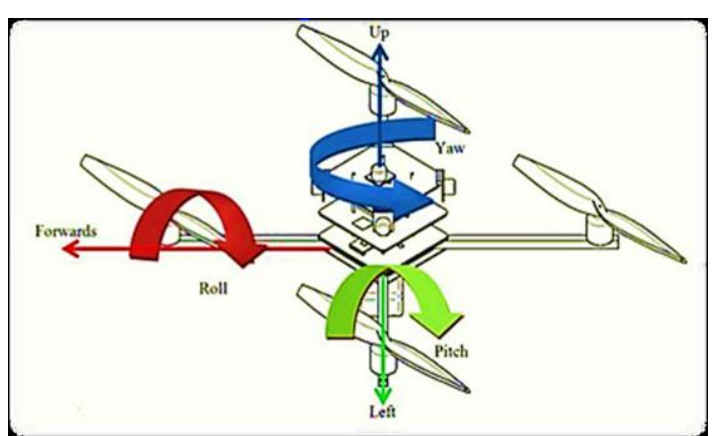

Fig. 1 Yaw Roll Pitch of Quadcopter

The quadcopter in Fig. 1 it's a flying object, which flies with a help by four propellers, Therefore, is so called. Two opposite propellers rotate in one direction, for take-off. First pair opposite propellers in Fig. 3 rotates in one direction for keeping balance in the axis. Second pair opposite propellers rotates in opposite direction, for keeping balance in the axis as in Fig. 4. The main reason for opposite rotations of opposite pairs it's the elimination a rotation the quadcopter in the $\mathrm{Z}$ axis.

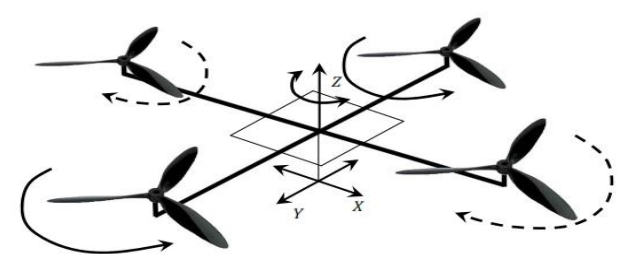

Fig. 2 Principle Function of Quadcopter

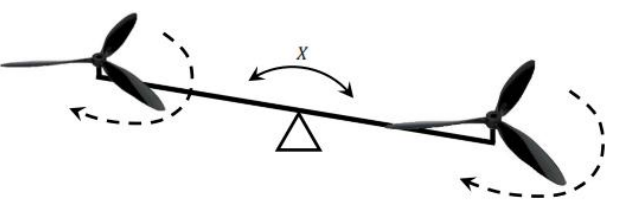

Fig. 3 Principle Function of First Arm

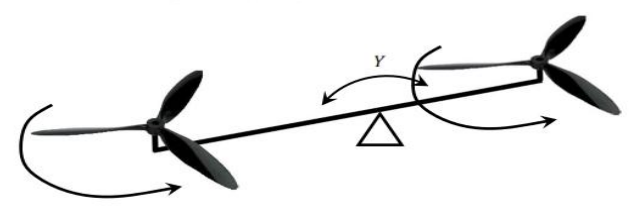

Fig.4 Principle Function of the Second Arm

\section{SYSTEM BLOCK DIAGRAM}

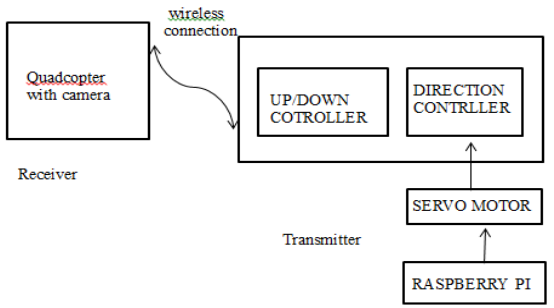

Fig. 5 System Block Diagram 
In the project I have use the IP webcam, with the help of the mobile phone. The output of the video is seen on the monitor or laptop with the help of the wireless network connection. It does not require any Internet to access it. It is connected with a network of Android web server and forming a connection using mobile hotspot connection. The same module can be done with a wireless video transmitter. I have use it since to save the cost of project. And for detection purpose raspberry pi is connected to controller unit in order to track it continuously.

The arrangement for the system is as shown in the Fig. 5. The working of the system is, the camera mounted on the quadcopter will continuously capture the video and send to the Raspberry pi. The Raspberry pi will try to find the object and track the object with the help of the servo motor connected to it. The working of the Raspberry pi in order to detect the object and track object is given in the next section.

\section{ECONTROLLING ARRANGEMENT AND ITS WORKING}

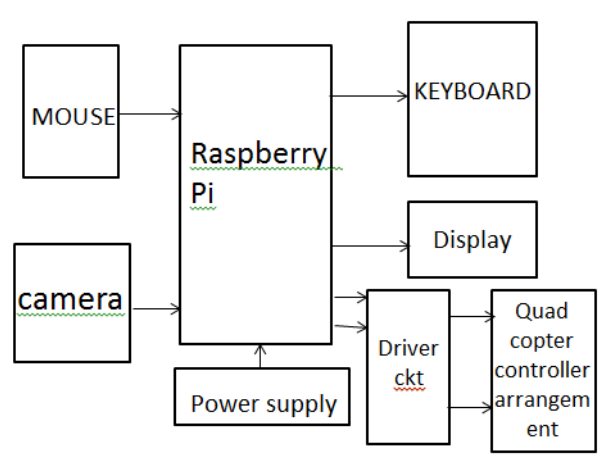

Fig. 6 Block Diagram of Controlling Arrangement With Raspberry Pi

The camera connected to Raspberry Pi will capture the image as shown in Fig. 6 and the image is given to Raspberry Pi for the processing that means it will first resize the image captured by the camera and convert the RGB image to HSV image. The HSV is nothing but the Hue, Saturation and Value as shown in flowchart below in Fig. 7. In this process the image is converted to black and white image. Then by using the condition for intensity values in image we obtained the binary image, that is if object present in specific region then ( Pixel > Threshold_Min \& Pixel ) = Pixel output image is 1 else it is zero. For this purpose we have two sliders to set upper and lower pixel values. It is done for all three components that is Hue, Saturation, Value. From this three condition we obtained the three different images. This images are Black and White images, which has region of object as $1^{\text {,s }}$ and intensity value greater or less than Threshold. The pixel that do not pass condition are zero. Then combining all the three images means Anding all the three images we get a single image. So output of this is, all the pixel which are white in three images will be white in output of this step. So these will be region to which will have 1 's but with lower areas and of random shapes. Now we use Hough Transform to detect object and this will draw marker on detected object with center and radius showing circle.

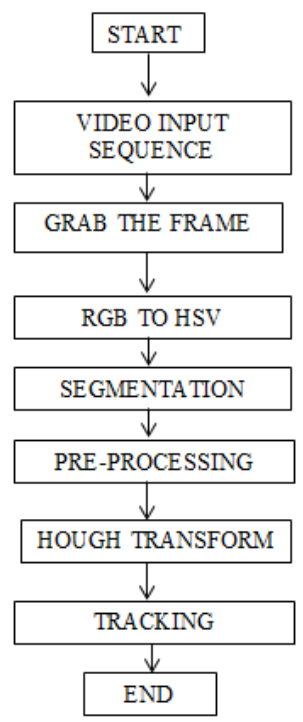

Fig. 7 Flowchart of Object Tracking 
It is a color-based algorithm for object tracking. Fig. 7 shows the flow chart of color-based object Tracking. The flowchart have different steps such as RGB to HSV conversion, segmentation, pre-processing process, hough transform etc. For every video input sequence, we grab the frame from video. The grab frame is RGB frame which converted into HSV frame. We segmented the HSV frame. The segmented frames are tracked in the successive frames. Then applied pre-processing process on segmented frame which contains morphological and filtering operation. Here Gaussian filter is applies to remove the noise and make the image smoother. After that hough transform applied on it to detect the object and mark the object with circle.

\section{ASELECTION OF CONTROLLER}

\section{System Hardware And Discription}

- Multicopter flight controller comparison by sensors

As we know, A flight controller is a circuit board that collect pilot's commands and sensors makes adjustment to keep your multicopter fly the right direction and hover in the air.Multicopter FC sensors comparison chart as below, field available then write "Y" or "Opt" (optional) otherwise "N". ( Gyro = Gyroscopes, Acc $=$ Accelerometer, DSL= Dynamic System Language, Temp $=$ Temperature )

Table 2 Comparison of Controller By Sensor

\begin{tabular}{|c|c|c|c|c|c|c|c|}
\hline Controllers & \multicolumn{9}{|c|}{ Sensors } \\
\hline FC & Gyro & Acc & GPS & Compass & Pressure & DSL & Temp \\
\hline APM2.8 & MPU-6000 & Y & Y & Y & Y & N & Y \\
\hline Openpilot CC3D & MPU-6000 & Y & Opt & Opt & Opt & N & Y \\
\hline Acro Naze32 & MPU6500 & Y & N & N & N & N & N \\
\hline KK2 & 6050MPU InvenSense & Y & N & N & N & N & N \\
\hline Naza-M Lite & 3-axis gyroscope & Y & Y & Y & Y & N & Y \\
\hline
\end{tabular}

- Multicopter flight controller comparison by Flight modes

Multicopter FC Flight modes comparison chart as below, field available then write "Y" otherwise "N". ( $\mathrm{HH}=$ Heading Hold, $\mathrm{ACC}=$ Accelerometer, $\mathrm{AH}=$ Auto Hover, $\mathrm{PH}=$ Position Hold FP = Fly By Click )

Table 3 Comparison of Controller By Flight Modes

\begin{tabular}{|c|c|c|c|c|c|c|}
\hline Controllers & \multicolumn{5}{|c|}{ Flight modes } \\
\hline FC & HH & ACC & AH & Height Hold & GPS PH & GPS FP \\
\hline APM2.8 & Y & Y & Y & Y & Y & Y \\
\hline Openpilot CC3D & Y & Y & Y & N & Y & N \\
\hline Acro Naze32 & Y & Y & Y & Y & Y & N \\
\hline KK2 & Y & Y & Y & N & N & N \\
\hline Naza-M Lite & Y & Y & Y & Y & Y & Y \\
\hline
\end{tabular}

\section{BINTRODUCTION TO SYSTEM}

The present system which I am developing is for disaster monitoring with a camera mounted on it which is operated through the Raspberry pi. Through this system I can detect the particular object. I am also providing a sensor in order to detect the explosive material called IED sensor. Depending on the availability of the IED sensor will be mounted on the UAV. In order to get the information of any vehicle which is travelling on the way we required to rely on Global Positioning System. The GPS provides the Global Positioning Information of vehicle, which relies on external source called satellites. But the satellite signal may get cut or have small signal strength in cluttered areas or is less reliable at low altitude areas. So I think of to use the Unmanned Aerial Vehicle, not only this by attaching the Raspberry pi which is a credit size single cheap computer in order to classify the object that we want.

It is a multifunctional UAV which will monitor and classify the object along with it also perform the explosive detection. It plays a great role in disaster occurred areas in order to detect the object and rescue application. It also plays a role in detecting the explosive material in a cluttered area. In a flooded area in order to monitor it is easy for UAV to move above the surface of the ground and water. And provides the detection as well as live streaming of the particular area and images can be captured by it. It has a sensor IED in order to detect the explosive material. It can provide the full image of the object by moving around the object. It is operated by remote control. At the transmitting side you can see the live streaming and images of the area on the display. You can also able to see the detected object with a sign indicating particular object. 


\section{TYPES OF UAV FRAME}

1) Tricopter:

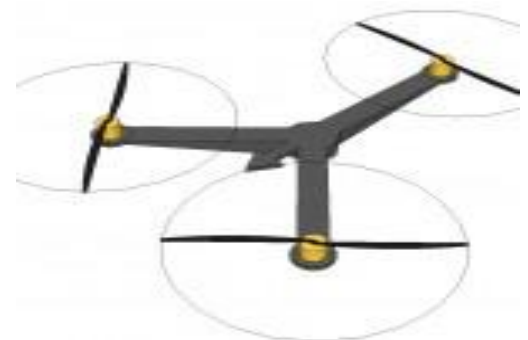

Fig.8 Tricopter

\section{Description}

A UAV which has three arms as shown in Fig. 8, each connected to one motor. The front of the UAV tends to be between two of the arms (Y3). The angle between the arms can vary, but tends to be 120 degrees. In order to move, the rear motor normally needs to be able to rotate (using a normal RC servo motor) in order to counteract the gyroscopic effect of an uneven number of rotors, as well as to change the yaw angle. A Y4 is slightly different in that it uses two motors mounted on the rear arm, which takes care of any gyroscopic effects - no servo is therefore needed.

\section{Advantages}

Different "look" for a UAV. Flies more like an airplane in forward motion. Price is theoretically lowest among those described here since it uses the fewest number of brushless motor (and ESC).

\section{Disadvantages}

Since the copter is not symmetric, the design uses a normal RC servo to rotate the rear motor and as such, the design is less straightforward than many other multi-rotors. The rear arm is more complex since a servo needs to be mounted along the axis. Most, though not all flight controllers support this configuration.

2)Quadcopter:

\section{Description}

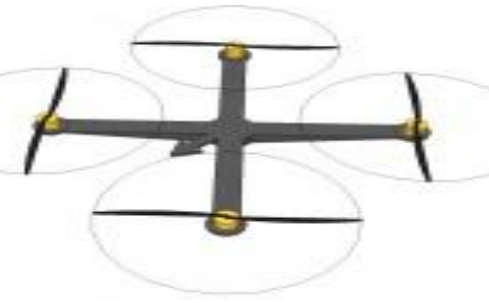

Fig. 9 Quadcopter

A "quadcopter" drone which has four arms as shown in Fig. 9, each connected to one motor. The front of the UAV tends to be between two arms (x configuration), but can also be along an arm (+ configuration).

\section{Advantage}

Most popular multi-rotor design, simplest construction and quite versatile. In the standard configuration, the arms / motors are symmetric about two axes. All flight controllers on the market can work with this multirotor design.

\section{Disadvantages}

There is no redundancy, so if there is a failure anywhere in the system, especially a motor or propeller, the craft is likely going to crash.

\section{3)Y6 Hexacopter:}

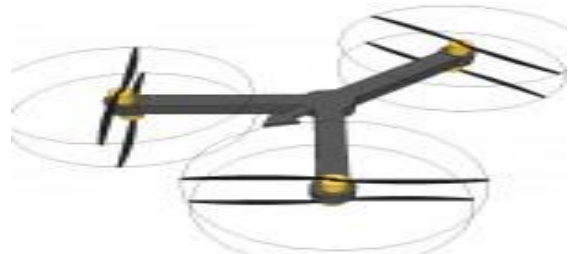

Fig. 10 Y6 Hexacopter 


\section{Description}

A Y6 design is a type of hexacopter but rather than six arms, it has three support arms, with a motor connected to either side of the arm (for a total of six motors) as shown in Fig. 10. Note that the propellers mounted to the underside still project the thrust downward.

Advantages

A Y6 design actually eliminates a support arm (as compared to a quadcopter), for a total of three. This means the copter can lift more payload as compared to a quadcopter, with fewer components than a normal hexacopter. A Y6 does not have the same issue as a Y3 as it eliminates the gyro effect using counter-rotating propellers. Also, should a motor fail, there is still a chance the copter can land rather than crash.

\section{Disadvantages}

This uses additional parts, so compared to a quadcopter which uses the same components, the equivalent hexacopter would be more expensive. Additional motors and parts add weight to the copter, so in order to get the same flight time as a quadcopter, the battery needs to be larger (higher capacity) as well. The thrust obtained in a Y6 as opposed to normal hexacopter is slightly lower (based on experience), likely because the thrust from the top propeller is affected by the lower propeller. Not all flight controllers support this configuration.

\section{Plan for Emergencies-}

Most emergencies result in the UAV crashing, and most crashes occur when you're just getting started in the field and/or with a new UAV. The list below is intended to give you things to think about and some ideas as to how to react and why for new pilots. This is case by case and in some situations the opposite of what is suggested here may be appropriate, so know yourself, know your environment and be safe! In almost all cases, it's better to lose the UAV than risk hitting people or property.

$>$ UAV is very far away and you don't know which way the UAV is facing.

- Throttle down and see if the UAV responds (testing to see if you still have communication).

- If the UAV responds to the throttle, try landing right there (if you can).

- If the spot is not good for a landing, gently try pitching the UAV forward (nothing drastic) and if the UAV responds based on yourviewpoint by moving left or right in the sky; this will help you determine its orientation. If it does not seem to go left or right, it may be moving towards or away from you, so undo what you just did and try yawing left.

$>$ UAVsuddenly veers off course drastically (with or without reason).

- If you're not skilled at piloting (to try to recover it), throttle down completely and let it crash: Making drastic corrections may cause you to lose sight of the UAV or worse, increase its altitude for a crash from a greater height.

$>$ UAV is quickly heading for people.

- Throttle up to max to have it go up and over them; your UAV is likely still be in the correct general orientation, so it's better to lose the UAV than to hit people.

- Once it's high enough in the sky, try your best to regain control and position it such that it's over a clear landing spot.

$>\mathrm{UAV}$ is heading quickly for you.

- Throttle down to zero; have it ram the ground as opposed to risking hitting your head.

$>$ UAV does not respond to input from the controller.

- Nothing you can do. If your UAV is still in the air, your transmitter's batteries may be dead.

$>$ UAV loses a propeller.

- Losing a propeller affects both thrust and yaw; try to correct for yaw first (you'll likely need to keep the joystick associated with yaw at an extreme position), and then simultaneously throttle up gently to try to prevent a crash, then throttle down.

\section{GENRAL BLOCK DIAGRAM OF A QUADCOPTER}

The brain of the Quadcopter is a microcontroller. The microcontroller used for the Quadcopter is KK2.1. The original KK gyro system has been updated to the incredibly sensitive 6050 MPU system making this the most stable KK board ever and adds the addition of an auto-level function. At the heart of the KK2.1 is the ATMEL Mega 644PA 8-bit AVR RISC-based microcontroller with 64k of memory. An additional header has been added for voltage detection, so now there is no need for on-board soldering. A handy piezo buzzer is also included with the board for audio warning when activating and deactivating the board, which can be supplemented with an LED for visual signaling. The following Fig. 11 shows you the general block diagram of Quadcopter with drivers and motors connected to it. The motor spins the propellers connected to it. The Fig. 12 shows the microcontroller that is the KK2.1 board which is used to operate the motors connected to it. 


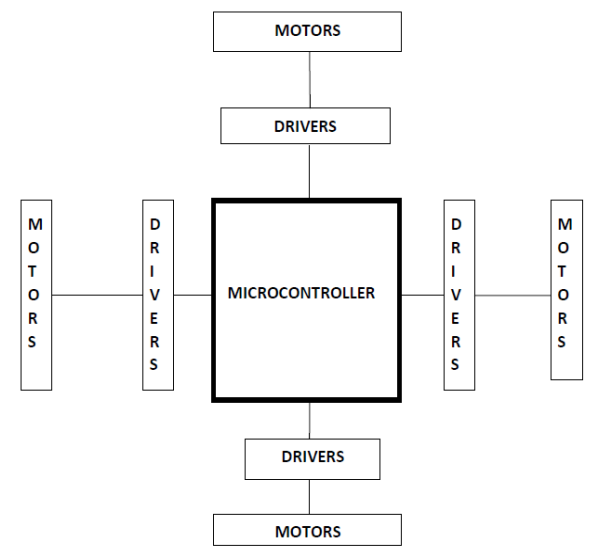

Fig. 11 General Block Diagram of Quadcopter

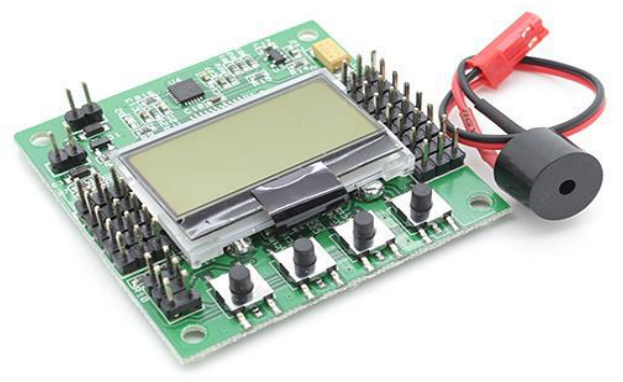

Fig. 12 KK2.1 Microcontroller Board

The KK2.1 Multi-Rotor controller manages the flight of (mostly) multi-rotor Aircraft (Tricopters, Quadcopters, Hexcopters etc). Its purpose is to stabilize the aircraft during flight and to do this, it takes signals from on-board gyroscopes (roll, pitch and yaw) and passes these signals to the Atmega324PA processor, which in-turn processes signals according the users selected firmware (e.g. Quadcopter) and passes the control signals to the installed Electronic Speed Controllers (ESCs) and the combination of these signals instructs the ESCs to make fine adjustments to the motors rotational speeds which in-turn stabilizes the craft.

KK2.1 Multi-Rotor control board also uses signals from your radio system via a receiver (Rx) and passes these signals together with stabilisation signals to the Atmega324PA IC via the aileron; elevator; throttle and rudder user demand inputs. Once processed, this information is sent to the ESCs which in turn adjust the rotational speed of each motor to control flight orientation (up, down, backwards, forwards, left, right, yaw).

KK2.1 has inbuilt Inertial Measurement Unit or IMU is the electronic sensor system of the quadcopter which measures velocity, orientation and gravitational forces of the quadcopter. It calculates the orientation of the quadcopter - the three orientation angles, Roll, Pitch and Yaw. These angles are then fed into some controlling electronics that uses those angles to calculate the required changes in the motor speeds. The IMU contains at least 6 sensors, referred to as 6DOF. [3] These sensors should be a 3-axis accelerometer and a 3-axis gyroscope. Sometimes another sensor, a 3-axis magnetometer, is added for better Yaw stability(totally 9DOF).

The accelerometer measures acceleration and force, so downward gravity can be measured too. It has 3 axis sensors, so the orientation can be worked upon also. A gyroscope measures angular velocity or rotational speed around the three axis. If accelerometer only is used then we can measure the orientation with reference to the surface of earth. Sometimes the accelerometer can be very sensitive and unstable because when the motor vibration is bad, the orientation is messed up. [2] Therefore a gyroscope is used as a solution to this problem. The gyroscope too drifts a lot. When the sensor rotates, the gyroscope will give the angular velocity. But when it stops rotating, it doesn't necessarily go back to $0 \mathrm{deg} / \mathrm{s}$. The gyroscope readings will provide an orientation that continues to move slowly (drifts) even when the sensor stops rotating. [3] Hence both the accelerator and the gyroscope sensors have to be used together to obtain a productive orientation. Unfortunately, the accelerometer cannot sense yaw rotation. [3] Hence a magnetometer is used for this purpose. A magnetometer is a device that measures the directions and strength of the magnetic field. It determines which way is south and north. The pole locations are then used as a reference together with the Yaw angular velocity around from the gyroscope, to calculate a stable Yaw angle. 
The components of Quadcopter are as follows

\section{Motor}

The motors you use will have a huge impact on the payload (or maximum load) which your UAV can support, as well as the flight time. We strongly suggest using the same (propulsion) motor everywhere. Note that even if a pair of motors are the same brand and model, and from the same production run, their speeds may vary slightly, which is something the flight controller will take care of.

KV

The KV rating / value of a motor relates to how fast it will rotate for a given voltage. For most multirotor aircraft, a low KV is desired (between 500 to 1000 for example) since this helps with stability. For acrobatic flight however, you might consider a KV between 1000 and 1500 and also consider using smaller diameter propellers. If the $\mathrm{KV}$ rating for a particular motor is $650 \mathrm{rpm} / \mathrm{V}$, then at $11.1 \mathrm{~V}$, the motor will be rotating at $11.1 \mathrm{~V} \mathrm{x} 650=7215 \mathrm{rpm}$. If you operate the motor at a lower voltage (say, $7.4 \mathrm{~V}$ ), the rpm will be $7.4 \mathrm{~V}$ $\mathrm{x} 650 \mathrm{rpm} / \mathrm{V}=4810 \mathrm{rpm}$. It is important to note that using a lower voltage tends to mean that the current draw will be higher $($ power $=$ current $\mathrm{x}$ voltage $)$.

\section{- Thrust}

Some brushless motor manufacturers give an indication of a motor's thrust corresponding to several propeller options (often presented in a table). The unit of thrust is often $\mathrm{Kg}$, Lbs or N. For example, if you are building a quadcopter and find that a specific motor can provide up to $0.5 \mathrm{Kg}$ of thrust with an 11 inch propeller, that means that four of these motors (with that given prop) can lift $0.5 \mathrm{Kg} * 4=2 \mathrm{Kg}$ at maximum thrust. Therefore when the quadcopter weighs just less than $2 \mathrm{Kg}$, it will only take off at maximum thrust. So we need to either choose a motor + propeller combination which can provide more thrust, or reduce the weight of the aircraft. If the propulsion system (all motors and props) can provide $2 \mathrm{Kg}$ of thrust (max) then your entire copter should be at most about half this weight $(1 \mathrm{Kg}$, including the weight of the motors themselves). The same calculation can be done for any given configuration. Let's assume a hexacopter's weight (including frame, motors, electronics, battery, accessories etc) to be $2.5 \mathrm{Kg}$. Each motor should therefore be capable of providing $(2.5 \mathrm{Kg} / 6 \mathrm{motors}) \mathrm{x}$ $2: 1=0.83 \mathrm{~kg}$ of thrust (or more).

\section{Propeller}

Propellers for multi-rotor aircraft are adapted from propellers used in RC airplanes. Why not use helicopter blades? Although it has been done, imagine the size of a hexacopter which used helicopter blades. Note that a helicopter-type system also requires that you vary the pitch of the blades which significantly adds to the mechanical complexity. I may also ask why not use a turbojet, turbofan, prop-jet etc? These are incredibly good at providing a lot of thrust, but also require a lot of power. If the objective of the drone was to move really fast rather than hover in confined areas, one of these may be a good option.

\section{ESC}

An ESC (acronym for "Electronic Speed Controller") is what allows the flight controller (covered in the next lesson) to control the speed and direction of a motor. The ESC must be able to handle the maximum current which the motor might consume, and be able to provide it at the right voltage. Most ESCs used in the hobby industry only allow the motor to rotate in one direction, though with the right firmware, they can operate in both directions.

\section{Battery}

Batteries used in UAVs are now almost exclusively Lithium polymer (LiPo), with some more exotic ones being Lithium-Manganese or other Lithium variations. Lead acid is simply not an option and $\mathrm{NiMh} / \mathrm{NiCd}$ are still too heavy for their capacity and often cannot provide the high discharge rates needed. LiPo offer high capacity with low weight, and high discharge rates. The downsides are their comparatively higher cost and continued safety issues.

\section{Voltage}

This battery's voltage should correspond with the motors we chose. Almost all batteries used these days are lithium-based and incorporate a number of $3.7 \mathrm{~V}$ cells, where $3.7 \mathrm{~V}=1 \mathrm{~S}$. Therefore a battery which is marked as $4 \mathrm{~S}$ is likely $4 \times 3.7 \mathrm{~V}=14.8 \mathrm{~V}$ nominal. Providing the number of cells however will help you determine which charger to use. A single cell high capacity battery may physically look a lot like a low capacity multi-cell battery. 


\section{Capacity}

A battery pack's capacity is measured in amp-hours (Ah). Small battery packs can be in the range of 0.1 Ah (100mAh) though battery packs for medium sized drones are 2-3Ah (2000mAh-3000mAh). The higher the capacity, the longer the flight time, but the heavier the pack will be. You can expect the flight time of a normal UAV to be in the order of 10-20 minutes, which might not seem like a long time, but you need to consider it's always fighting against gravity, and unlike an airplane, there are no surfaces to help with lift.

\section{Discharge Rate}

The discharge rate from a lithium battery is measured in $\mathrm{C}$, where $1 \mathrm{C}$ is the capacity of the battery (normally in amp hours unless you're looking at a very small drone the size of your hand). The discharge rate of most LiPo batteries is at least 5C (five times the capacity), but since most motors used in multirotors consume high current, the battery needs to be able to discharge at incredibly high current, which is often in the order of $30 \mathrm{~V}$ or more.

\section{Standard RC Transmitter}

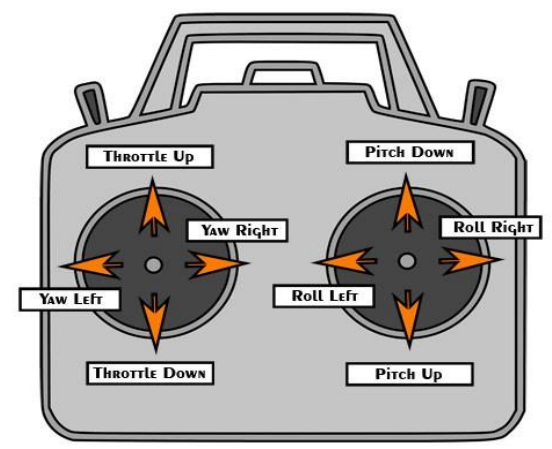

Fig. 13 Standard RC Transmitter

In order to control the quadcopter the joy stick is used. To control or balance or change the direction the Yaw, Roll, Pitch and Throttle is used. It is also shown in table no.1. AFHDS2A-The acronym for it is Automatic Frequency Hopping digital System 2A.Due to Frequency hopping technique it has long battery life experience. It is $2.4 \mathrm{GHz}$ system having built in channel coding, error correction, reduce error ratio, reliable Transmitter. The specification for the Transmitter used in the project is as follow,

Number of Channels - 6

Resolution - 1024 i.e, $2^{10}$

System type - AFHDS2A

Modulation Technique - GFSK

The specification for Receiver are as follow,

RF range -2.4055 to $2.475 \mathrm{GHz}$

Channel Bandwidth $-500 \mathrm{KHz}$

Number of Channels - 140

RF Power - less than $20 \mathrm{dbm}$

Modulation Technique - GFSK

Antenna - Dual Antenna (26mm*2)

Receiver Sensitivity - $-105 \mathrm{dbm}$

E OPENCV PROGRAMMING

This literature was write by Vadim Pisarevsky, the Senior Software Engineer of Intel Corporation, Software and Solutions Group. This literature introduce general information of OpenCV, Getting Started with OpenCV, Modules Description, Interaction with Intel IPP, Python Interface, Some Usage Examples and Summary. What is OpenCV? OpenCV (Open Source Computer Vision Library) is a library of programming functions mainly direct at real time computer vision, open up by Intel, and now stand by Itseez and Willow Garage. Free for use under the open source BSD license. The library is cross-platform. It is central mainly on real time image processing. If the library finds Intel's Integrated Performance Primitives on the system, it will use these proprietary optimized routines to accelerate itself. Why we need OpenCV? Now, computer Vision Market is large and still growing. But there is no standard API (like OpenGL and DirectX in graphics, or OpenSSL in cryptography), most of CV software is of 3 kinds:

1. Research code (slow, unstable, independent/incompatible data types for every library/toolbox)

2. Very expensive commercial toolkits (like Halcon, MATLAB + Simulink, ...) 
3. Specialized solutions bundled with hardware (Video surveillanceManufacturing control systems, Medical equipment ...)

So, we need a standard library that simplifies development of new program and solutions much easier. So, Intel develop OpenCV, and Intel optimization for Intel Platforms that creates new usage models by achieving real-time performance for quite"heavy" algorithms and makesOpenCV (Open Source Computer Vision Library) is a library of programming functions mainly direct at real time computer vision, open up by Intel, and now stand by Itseez an Willow Garage. Free for use under the open source BSD license. The library is cross-platform. It is central mainly on real time image processing. If the library finds Intel's Integrated Performance Primitives on the system, it will use these proprietary optimized routines to accelerate itself.

OpenCV is written in C++ and its primary interface is in C++, but it still retains a less comprehensive though extensive older $\mathrm{C}$ interface. There are now full interfaces in Python, Java and MATLAB. The API for these interfaces can be found in the online documentation. Wrappers in other languages such as C\#, Ruby have been developed to encourage adoption by a wider audience. All of the new developments and algorithms in OpenCV are now developed in the $\mathrm{C}++$ interface. In this project I have work on Rispberry pi learn first how to use the Rispberry pi. In order to use the Rispberry pi I need to learn the operating system for it. The main objective of my UAV to detect the object for that purpose I have written the Motion Detection Code. I also studied the various command to operate the Rispberry pi.

\section{A INTRODUCTION}

\section{Result And Discussion}

Quadcopters or drones first came to application as small toys, or school/university projects and then no sooner began to garner widespread attention- used in big-budget movies, photography of high profile sports, agricultural use to rectify lands and detect levels of pesticides as well as other components like nitrogen in plants, search and rescue, land mapping, military, etc. The commercial as well as private use of drones is enlarging. The main point is that with growing progress in technology, drones too are coming in different shapes, sizes and configuration (quadcopters, hexacopters, etc.) for better load and yaw stability. Moreover, extended components like camera, water- resistant components or GPS tracker make it easy in missions of combating, surveillance and especially search and rescue. After 10 years, the market for commercial drones will reach $\$ 1.7$ billion. Each year, $\$ 6.4$ billion is being spent developing drone technology. The drones are even providing new job opportunities. 70,000 new drone-related jobs are projected within the next three years in USA alone. 100,000 new jobs are expected till 10 years. Moreover, schools are offering drone degrees \& programs in order to provide a trained workforce capable of meeting this demand. This may also prove that there is no need for people to fear about losing their jobs because of replacement by robots or drones.

But there are also some concerns like all the new leading technologies. It is important to plan appropriately to achieve a productive outcome. Privacy among being the major concern in warfares, could be used as a means of spying which is mere exploitation and negative use of quadcopter technology. Since the advantages of quadcopter technology are more than its demerits (are very well rectifiable), then according to drone experts, drones will be "in trend" within next 10 to 20 years. There is a wide scope that with its extending use in almost every field and with greater powerful components, drones will surely come into full time existence. Hence quadcopters will very soon start taking over larger roles in a variety of jobs. The Fig. 14 and Fig. 15. shows the color detection and setup for the controlling direction with the help of motor and Raspberry pi connected to Ardino.
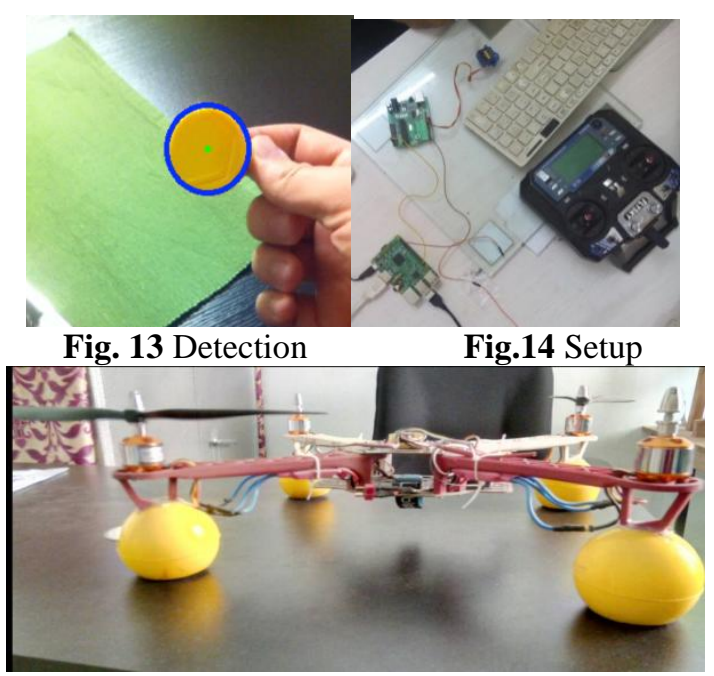

Fig. 15 Quadcopter with KK2.1 Controller 
The Above image in Fig. 15 shows the quadcopter with KK2.1 Controller which is used in the project. Some live images taken with the help of IP Webcam are also shown below in Fig. 16.

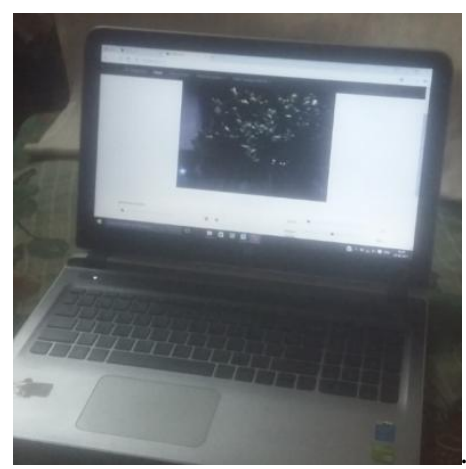

Fig. 16 Image captured by IP Webcam

The Results that I got are accurate as per the coded programme but due to mechanical coupling to the joystick controller makes it somewhat less accurate.

\section{Conclusion}

Drones will soon take on be an imperative existence in the coming future. They will be seen taking up larger roles for a variety of jobs including business in the immediate future They could become a part of our daily lives, from smallest details like delivering groceries to changing the way farmers manage their crops to revolutionizing private security, or maybe even aerial advertising. Today, quadcopters are capturing news video, recording vacation travel logs, filming movies, providing disaster relief, surveying real estate and delivering packages. They are categorized according to their corresponding uses. Some are for military purposes provided with missiles and bombs, some for surveillance and reconnaissance purposes. The Aim of the Project is satisfied and satisfactory results are obtained.

\section{Refrences}

[1] Scheve, T. (n.d.). Howstuffworks. Accessed October 30, 2012, from Discovery: http://science.howstuffworks.com/reaper1.htm

[2] http://blog.oscarliang.net/build-a-quadcopter-beginners-tutorial-1/- Posted on June 252013.

[3] The UAV Accessed October 27, 2012

[4] http://www.theuav.com/

[5] Finn, W. W. (2011, 7 4). Global Race on to Match U.S Drone Capabilities. Retrieved from The Washington Post: http://www.washingtonpost.com/world/national-security/global-race-on-tomatch-usdronecapabilities/2011/06/30/gHQACWdmxH_story.html

[6] http://www.philforhumanity.com/Drones.html

[7] AeroVironment's Global Observer: Flying High, Again, April 06, 2011, Accessed October 27, 2012 from Defense Industry Dailyhttp://www.defenseindustrydaily.com/aerovironments-global-observer-flying-high-again- 03902/

[8] Sinha, Ashish, Jubilant in JV with Aeronautics for UAVs, September 27, 2010, Accessed October 26, 2012 http://www.financialexpress.com/news/jubilant-in-jv-with-aeronautics-for-uavs/688501/0

[9] 2013 Undergraduate Students Unmanned Aerial Systems Competition AUVSI Draft RFP v5.0, Accessed October 26, 2012

[10] www.farmingdrones.com

[11] www.abcnews.com

\footnotetext{
IOSR Journal of Electrical and Electronics Engineering (IOSR-JEEE) is UGC approved Journal with Sl. No. 4198, Journal no. 45125.

Mr.Mayur S. Malokar. "Visual Control Of Unmanned Aerial Vehicle For Handling Disaster Manangement Issue." IOSR Journal of Electrical and Electronics Engineering (IOSR-JEEE) 12.4 (2017): 06-19.
} 